\title{
Roy Compte, La figure du handicap. Analyse d'une construction sociale et politique
}

Thèse de Doctorat de l'Université Paul Valéry, Montpellier III, présentée en sociologie par M. Roy Compte, sous la direction de M. le Professeur Jean-Marie Brohm

Gilles Bui-Xuân

\section{OpenEdition}

1 Journals

Édition électronique

URL : http://journals.openedition.org/corpsetculture/659

DOI : 10.4000/corpsetculture.659

ISSN : 1777-5337

Éditeur

Association Corps et Culture

Édition imprimée

Date de publication : 1 juin 1999

ISSN : 1268-5631

Référence électronique

Gilles Bui-Xuân, « Roy Compte, La figure du handicap. Analyse d'une construction sociale et politique », Corps et culture [En ligne], Numéro 4 | 1999, mis en ligne le 25 avril 2007, consulté le 22 septembre 2020. URL : http://journals.openedition.org/corpsetculture/659 ; DOI : https://doi.org/10.4000/ corpsetculture.659

Ce document a été généré automatiquement le 22 septembre 2020.

(c) tous droits réservés 


\section{Roy Compte, La figure du handicap. Analyse d'une construction sociale et politique}

Thèse de Doctorat de l'Université Paul Valéry, Montpellier III, présentée en sociologie par M. Roy Compte, sous la direction de M. le Professeur Jean-Marie Brohm

\section{Gilles Bui-Xuân}

\section{NOTE DE L'ÉDITEUR}

Le Jury est composé de :

Albert Restoin, professeur en Sciences de l'Éducation, Université de Montpellier 1.

Michel Chauvière, Directeur de recherche au CNRS, Groupe d'analyse des politiques publiques, Paris.

Gilles Bui-Xuân, Maître de conférences, Université de Montpellier 1, Jean-Marie Brohm, Professeur de Sociologie, Université Paul Valéry, Montpellier 3.

1 Il faut tout d'abord souligner que l'itinéraire de M. Roy Compte n'est pas celui d'un étudiant commun. Une trentaine d'années passée dans l'enfance inadaptée, d'abord comme éducateur, puis comme professeur d'Éducation Physique et Sportive, d'une part, un engagement comme dirigeant d'associations (il est encore vice-président de la Fédération Française du Sport Adapté) d'autre part, l'ont amené à s'interroger sur la nature de sa propre intervention, et bien au-delà, sur l'essence même d'un concept récent : le handicap.

2 Son ambition n'était-elle pas de contribuer au développement d'une sociologie du regard? En effet, à travers l'analyse de l'image que renvoie la personne handicapée, c'est une construction sociale et politique qu'il s'agit de décrypter, à l'origine de représentations évolutives mais parfaitement repérables dans le temps, mais aussi initiatrice d'un discours qui va déterminer l'action sociale. Il est vrai que le regard de 
M. Roy Compte n'est pas neutre, car non seulement il est professionnellement impliqué, mais aussi parce qu'il est celui d'un artiste, d'un peintre, qui plus est, figuratif. Il aurait voulu éduquer notre regard; il l'aura tout au moins orienté, tant il est vrai qu'après la lecture de cette thèse il semble difficile de voir le handicap comme avant.

3 Partant d'une étude presque exhaustive de la littérature sur la question, l'auteur se questionne sur l'évolution récente que la société, ou plutôt notre société, entretient visà-vis des personnes handicapées. Il émet l'hypothèse que leur prise en charge toujours plus importante serait à l'origine de leur marginalisation. En effet, plus l'intervention sociale se développerait en leur faveur, et plus la représentation commune du handicapé serait négative. Au point où l'on pourrait se demander si l'effort que déploierait la société pour les faire accéder à la normalité ne tuerait pas paradoxalement toute acceptation de l'altérité.

4 Pour vérifier ces hypothèses, l'auteur développe toute une panoplie de méthodes, complémentaires, croisant méthodes quantitatives et qualitatives, qu'il applique à des populations diverses, allant de personnes non concernées, aux structures de travail, en passant par les travailleurs sociaux, mais surtout, ce qui fait l'originalité de la thèse, par une interpellation des personnes handicapées elles-mêmes, notamment déficientes mentales. Il complétera ses données émanant d'entretiens ou de questionnaires par une analyse documentaire impressionnante.

5 Évidemment, il n'est guère possible d'étudier un tel phénomène sans se référer à l'histoire, que M. Roy Compte ne brosse pas de façon événementielle, mais en la confrontant sans cesse à la gestion de la norme et/ou de l'altérité.

6 Le handicap est alors perçu comme phénomène social total, en tout point créé par le social, mais en retour faisant ou défaisant le social dans ses rapports à l'intégration et à l'exclusion. Aujourd'hui, il appelle à une figuration des acteurs sociaux qui doivent impérativement sauver la face dès qu'ils sont confrontés au handicap.

7 D'abord sous la férule des aliénistes, il implique l'internement asilaire.

8 Il revêt ensuite les habits de l'infirme avant d'être traduit en handicap. Mais s'il passe de réactions de charité à celles d'assistance, puis de solidarité, il pose toujours le problème du rapport à la norme, celui de la gestion de l'altérité, pour toujours se terminer dans l'exclusion. On s'aperçoit en fait que l'universalité du handicap confère au « handicapé » une identité dans le regard de l'autre, qui a peur d'être touché dans son intégrité. Et si la charité est individuelle, renvoyant à une culpabilité fantasmatique, à soi et à sa propre monstruosité, la solidarité, elle, est sociale et politique, façonnée par un monde qui reflète notre propre image : elle est chose sociale.

9 La première enquête se rapporte à un public non concerné. Roy Compte fait passer un questionnaire à 81 sujets, qu'il étaye d'entretiens individuels.

10 Les résultats révèlent que dans l'ensemble le handicap renvoie toujours à des valeurs négatives. Mais si $64 \%$ des personnes interrogées ont pensé spontanément au handicap physique, c'est pourtant le handicap mental qui constitue la figure fondamentale du handicap. Ainsi l'intégration de la figure du handicap dans l'ordre symbolique participe à sa légitimation dans l'ordre social.

11 C'est pourtant le handicap mental qui présente «l'élément le plus handicapant de la condition handicapée », car en réalité, c'est celui qu'on « voit » le plus, ou plutôt qui se remarque le plus en renvoyant inéluctablement à une dangerosité. Mais au-delà de 
cette peur n'y a-t-il pas un sentiment de culpabilité ? Et derrière cette culpabilité, l'angoisse de ne pouvoir faire face à « cette inquiétante étrangeté » ?

12 La deuxième enquête s'intéresse aux travailleurs sociaux qui représentent, d'après l'auteur, les rouages de la violence symbolique exercée par l'État et ses institutions. 146 questionnaires sont dépouillés.

13 Pour ces professionnels le handicap physique évoque courage et injustice, mais aussi volonté, alors que le handicap mental n'inspire que pitié et compassion. On retrouve donc ici les mêmes représentations que dans le grand public, et il s'avère que ces représentations n'évoluent guère avec l'ancienneté dans la profession. Elles sont toutefois nuancées, en ce qui concerne les handicapés mentaux, en fonction de la nature de l'implication professionnelle.

14 Une troisième enquête conduit à explorer le domaine du travail et de l'emploi. 40 entreprises et 60 C.A.T. ont répondu à un questionnaire et participé à des entretiens semi-directifs.

15 L'État providence va conduire peu à peu de l'infirme de guerre, à une extension au mutilé du travail, puis au travailleur handicapé, relevant d'un principe de solidarité nationale. Mais l'enquête révèle que les entreprises les ignorent le plus souvent, ou tout le moins payent leur tribut pour s'en dédouaner. En tout cas les personnes handicapées ne travaillent que rarement en faisant valoir leurs compétences. Ainsi, la reconnaissance de travailleur handicapé induit de nombreux effets pervers, tant chez les employeurs qu'auprès des demandeurs d'emploi.

16 Quant à l'intégration scolaire, elle ne concerne réellement que $7 \%$ des enfants et adolescents handicapés, $93 \%$ d'entre eux dépendant d'un enseignement spécialisé. Et pourtant ce n'est pas faute d'effort de la part de l'Éducation nationale qui, en réalisant une intégration en classes ordinaires, ferait réaliser des économies substantielles à l'État.

17 C'est que les représentations sociales sont tenaces, et que l'effort de redéfinition des classifications des handicaps a bien du mal à transformer les pratiques.

18 L'auteur montre d'ailleurs que l'aide sociale est toujours soumise à des enjeux de pouvoir, et que dans la période actuelle, le type de handicap a de moins en moins d'importance au fur et à mesure que montent chômage, pauvreté et exclusion. Ainsi, même si le nombre de jeunes handicapés diminue légèrement, celui des adultes handicapés augmente considérablement. C'est qu'il n'y a pratiquement plus de honte à se déclarer handicapé, d'autant moins de honte que la recherche d'un statut d'assisté organise la vie sociale d'une nouvelle catégorie de population : les « ayant droit ».

19 C'est dans ce contexte que l'ensemble des textes de loi concernant le handicap sont analysés, révélant le plus souvent l'opposition entre les tenants de la santé (les psychiatres) et les défenseurs de l'Éducation nationale.

20 Mais le travail aurait été incomplet s'il n'avait abordé le corps du handicapé, corps sans nom et sans visage, figure de l'imaginaire social. Car c'est d'abord la vision du corps du handicapé qui génère l'angoisse en nous renvoyant à l'image de notre propre corps, ce qui provoque immanquablement une gêne: fragile condition humaine! Et pourtant l'espoir que la recherche en génétique arrivera à venir à bout de toute tare à tendance à progressivement déculpabiliser et déresponsabiliser tant les individus que la société.

21 La sexualité est également convoquée. Une quatrième enquête permet de comparer les positions des travailleurs sociaux, quotidiennement confrontés à cette question, et 
celles des personnes handicapées elles-mêmes. Les professionnels sont dans l'ensemble plutôt favorables à l'épanouissement d'une sexualité qui n'aboutirait pas à l'enfantement. Ils ne craignent pas de préconiser la stérilisation des personnes handicapées mentales consentantes.

Quant aux personnes concernées, elles revendiquent toutes une sexualité normale, bien que chacune à leur manière, elles soient conscientes des difficultés qui l'entravent. Si elles sont handicapées physiquement le désir d'enfants n'est pas atteint alors qu'elles souffrent de leur isolement social. Si elles sont handicapées mentalement, l'enfantement semble difficile, voire irresponsable, alors qu'elles ne sont pas affligées par un isolement social.

Restait le sport, qui donne lieu à une cinquième enquête à partir du rassemblement d'un volume considérable d'articles de presse, portant sur le sport pour personnes handicapées au cours de plusieurs années. Elle révèle les contradictions d'un système qui adopte les valeurs d'un sport valide, contre l'avis des politiques et des théoriciens. C'est que le sport répond avant tout à la logique de ses dirigeants...

Ainsi, la thèse montre que la figure du handicap révèle la pâle figure du social, incapable d'imposer ses normes à tous. Par son incapacité à accepter l'autre comme tel, il marque son impuissance à s'accepter lui-même.

25 La thèse est stimulante. Elle se fonde sur un énorme travail de documentation, mais aussi sur une investigation de terrain, armée d'outils méthodologiques appropriés. L'analyse est fine et pertinente, parfois un peu redondante, mais avançant toujours de nouvelles données qui viennent compléter les précédentes pour mieux chasser les idées reçues. Ce tour de la question, abordé de façon originale par une approche figurative, ne peut laisser indifférent quiconque s'intéresse au handicap, d'autant qu'est posée in fine la question : mais qu'y a-t-il donc d'humain dans un handicapé mental profond ? Or, c'est bien ce dernier rempart d'humanité qui fonde justement la construction sociale et politique du handicap, quand sa négation conduirait inévitablement à «l'épuration".

À la suite d'une soutenance stimulante, M. Roy Compte obtient le titre de Docteur de l'Université Paul Valéry, et la mention «Très honorable avec les félicitations du Jury ». 